\section{Review: eye movement desensitisation and reprocessing is not better than exposure therapies for anxiety or trauma} Davidson PR, Parker KC. Eye movement desensitization and reprocessing (EMDR): a meta-analysis. J Consult Clin Psychol
2001 Apr;69:305-16.

QUESTION: Is eye movement desensitisation and reprocessing (EMDR) effective for trauma or anxiety? Do the presence of eye movements, therapist training, or disorder influence effectiveness?

\section{Data sources}

Studies were identified by searching PsycINFO and Medline (1988 to April 2000) and Current Contents (1997 to March 2000) and by scanning reference lists.

\section{Study selection}

Published studies were selected if they reported a clear unconfounded examination of the effect of EMDR in treatment experiments. Articles given only at conferences were excluded.

\section{Data extraction}

Data were extracted on the intervention, type of control, type of population, type of measure, and outcomes. Median effect sizes were calculated; only data collected immediately after treatment were used.

\section{Main results}

34 studies were included (33 randomised controlled trials). Studies were categorised according to type of outcome measure or comparison. Outcome categories were psychometric measures of post-traumatic stress disorder (PTSD), other psychometric measures, physiological measures, behavioural measures (including behavioural avoidance), and other measures. Comparison categories were no treatment, in vivo exposure or cognitive behavioural therapy (CBT), exposure-not in vivo, eyes fixed desensitisation reprocessing movements, other dismantling designs (EMDR procedures were used except that 1 aspect [not eye movements] was changed), and non-specific treatments. An effect existed for comparison type $(\mathrm{p}<0.01)$ but not for outcome measure; the outcome measures were collapsed by taking the median effect size for each study. EMDR was more effective than no treatment (13 studies) and non-specific treatment ( 5 studies) (table). EMDR was not more effective than exposure (not in vivo) (3 studies), in vivo exposure or CBT (3 studies), EMDR without eye movements (9 studies), or other dismantling designs (4 studies) (table). Results were similar when studies using therapists not trained by the EMDR Institute were excluded or when studies were grouped by treatment population (PTSD, traumatic memories, other anxiety disorders, or normal).

\section{Conclusions}

Eye movement desensitisation and reprocessing is more effective for anxiety or trauma than no treatment or therapies that do not use exposure to anxiety provoking stimuli. Eye movements are not necessary for the treatment effect, and therapist training or populations examined do not influence outcome.

†Not statistically significant.
Eye movement desensitisation and reprocessing (EMDR) for anxiety and trauma

\begin{tabular}{lcc} 
Control group & $\begin{array}{l}\text { Number of } \\
\text { comparisons }\end{array}$ & $\begin{array}{l}\text { Rosenthal's r } \\
(95 \% \mathbf{C l})^{*}\end{array}$ \\
No treatment & 13 & $0.44(0.31$ to 0.55$)$ \\
\hline Non-specific treatment & 5 & $0.40(0.18$ to 0.58$)$ \\
\hline Exposure (not in vivo) & 3 & $0.19(-0.12$ to 0.47$) \dagger$ \\
\hline In vivo exposure or cognitive behavioural therapy & 3 & $-0.28(-0.54$ to 0.02$) \dagger$ \\
\hline EMDR without eye movements & 9 & $0.10(-0.08$ to 0.27$) \dagger$ \\
\hline Other dismantling designs & 4 & $0.00(-0.26$ to 0.26$) \dagger$
\end{tabular}

*Positive values show benefit for EMDR.

\section{COMMENTARY}

EMDR had controversial origins as eye movement desensitisation (EMD). Early reports on EMD suggested that 1 session of treatment produced complete remission in PTSD. The treatment was trademarked. "Approved" training required participants to sign an undertaking that they would not train others. The treatment was elaborated to include 8 elements (mostly recognisable from CBT) and rebranded as EMDR. Longer treatments were advocated (a reversal of the trend in CBT). Unusual marketing strategies merged with apparently irregular scientific practices. ${ }^{1}$ Notably, EMDR has no coherent theoretical basis.

Controversy has continued with apparently conflicting meta-analyses and commentary. ${ }^{2}$ Davidson and Parker's well done meta-analysis manages convincingly to address 3 of the 4 key issues relevant to clinical science. (1) EMDR is found to be better than no treatment or placebo but no different than exposure. As a bonus, they found that the early reports of large effect sizes probably resulted from the use of process variables targeted directly in treatment sessions being used as primary outcome variables. (2) The training of therapists (whether or not it is approved by the EMDR franchising organisation) had no effect. It had previously been asserted that "treatment fidelity" was compromised in those studies that did not find positive results for EMDR. (3) The all important element of eye movements was found to be completely unimportant. The fourth question (whether evidence exists of specificity of treatment effects to disorders) was addressed, but unconvincingly given the number of studies and the way in which the analysis is conducted (in 4 categories rather than by specific disorder). If eye movements are not relevant to EMDR, then what remains is desensitisation and reprocessing-that is, CBT. EMDR has been assiduously marketed as a novel and effective treatment.

This meta-analysis, which is notable for the care with which it seeks to address the likely critique of those who advocate EMDR, firmly establishes that what is novel is not effective, and what is effective is not novel. Clinicians may wish to consider that the impressive paraphernalia of treatments such as EMDR is not, in the long run, a substitute for rapidly developing empirically grounded clinical interventions. ${ }^{3}$

Paul Salkovskis, MPhil, PhD, C Clin Psychol, FBPsS Institute of Psychiatry London, UK

1 McNally RJ. EMDR and Mesmerism: a comparative historical analysis. J Anxiety Disord 1999;13:225-36.

2 Rosen GM, Lohr JM, McNally RJ, et al. Power therapies, miraculous claims, and the cures that fail. Behavioural and Cognitive Psychotherapy 1998;26:99-101.

3 Ehlers A, Clark DM. A cognitive model of posttraumatic stress disorder. Behav Res Ther 2000;38:319-45 